\title{
Sensitivity of survival to migration routes used by juvenile Chinook salmon to negotiate the Sacramento-San Joaquin River Delta
}

\author{
Russell W. Perry • Patricia L. Brandes • \\ Jon R. Burau • A. Peter Klimley • \\ Bruce MacFarlane • Cyril Michel • John R. Skalski
}

Received: 28 February 2011 / Accepted: 24 January 2012

(C) Springer Science+Business Media B.V. 2012

\begin{abstract}
Populations of juvenile salmon emigrating from natal rivers to the ocean must often traverse different migratory pathways that may influence survival. In regulated rivers, migration routes may consist of a network of channels such as in the Sacramento-San Joaquin River Delta, or of different passage structures at hydroelectric dams (e.g., turbines or spillways). To increase overall survival, management actions in such systems often focus on altering the migration routing of fish to divert them away from low-survival routes and towards high-survival routes. Here, we use a 3-year data set of route-specific survival and movement of juvenile Chinook salmon in the Sacramento-San Joaquin Delta to quantify the sensitivity of survival to changes in migration routing at two major river junctions in the Sacramento River. Our analysis revealed that changes in
\end{abstract}

R. W. Perry · J. R. Skalski

School of Aquatic and Fishery Sciences,

University of Washington,

Seattle, WA 98103, USA

P. L. Brandes

US Fish and Wildlife Service,

4001 N. Wilson Way,

Stockton, CA 95205, USA

J. R. Burau

US Geological Survey, California Water Sciences Center, 6000 J Street,

Sacramento, CA 95819, USA overall survival in response to migration routing at one river junction depended not only differences in survival among alternative routes, but also on migration routing at the other river junction. Diverting fish away from a low-survival route at the downstream river junction increased population survival by less than expected, given the difference in survival among routes, because part of the population used an alternative migration route at the upstream river junction. We also show that management actions that influence only migration routing will likely increase survival by less than actions that alter both migration routing and route-specific survival. Our analysis provides an analytical framework to help fisheries managers quantify the suite of management actions likely to maximize increases in population level survival.

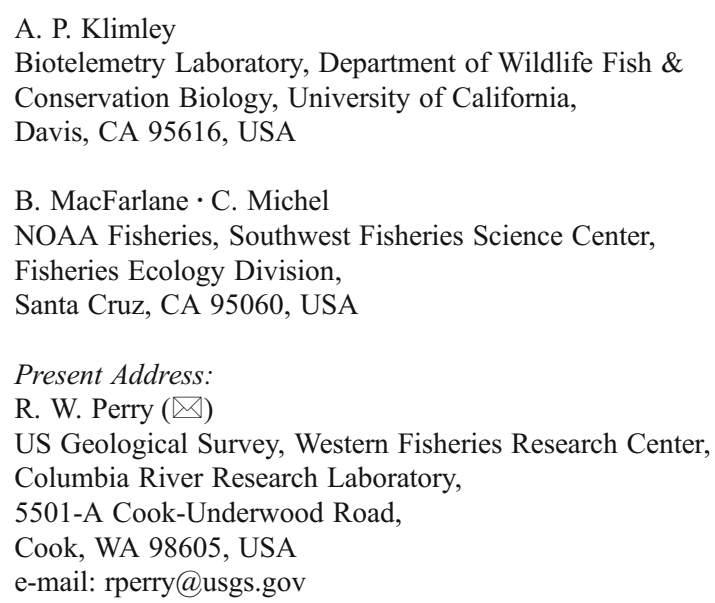


Keywords Migration · Telemetry · Juvenile salmon · Sacramento-San Joaquin Delta $\cdot$ Survival

\section{Introduction}

Population dynamics of migrating fish depend on how they use space over time. Populations may traverse different migratory pathways en route to their final destination. For example, variation in ocean currents may affect migration pathways of adult salmon returning to their natal rivers (Bracis 2010). In regulated rivers, migrating juvenile salmon may negotiate dams via alternative pathways such as spillways or turbines (Skalski et al. 2002, 2009). In estuaries and river deltas, complex channel networks offer an array of possible migration routes (Perry et al. 2010). In each of these examples, survival rates may vary among migration routes due to differences in migration timing, food resources, environmental conditions, or predator abundance. Thus, understanding variation in survival among migration routes can provide important insights about population dynamics.

The Sacramento-San Joaquin River Delta (hereafter, the Delta) is a complex network of natural and man-made channels through which juvenile salmon must navigate on their journey to the ocean (Fig. 1). As juvenile salmon enter the Delta from natal streams, they disperse among the Delta's complex channel network. This dispersal process is driven by the relative quantities of discharge entering each channel, the horizontal distribution of fish in the water column as they pass a channel junction (a main channel splitting into two or more channels), and by tidal cycles that alter flow patterns at river junctions. Once fish enter a given channel, they are subject to channel-specific processes that affect their rate of migration, vulnerability to predation, feeding success, growth rates, and ultimately, survival. Eventually, alternative migration routes converge at the exit of the Delta and the population once again comes together to migrate through San Francisco Bay.

Movement of juvenile salmon among migration pathways in the Delta is influenced by water management actions that route water from the Sacramento and San Joaquin Rivers into pumping stations in the southern Delta. In this paper, we focus on the influence of water management actions on juvenile salmon emigrating from the Sacramento River. Specifically, the
Delta Cross Channel is a man-made gated channel that diverts water from the Sacramento River into the interior Delta, where it then flows towards the pumping stations to be exported for agricultural and domestic uses (Fig. 1). Juvenile salmon entering the interior Delta exhibit lower survival probabilities than other migration routes, presumably due to longer migration times, entrainment at the pumping stations, and exposure to predators (Brandes and McLain 2001; Newman and Brandes 2010; Perry 2010). Furthermore, overall survival through the Delta (the fraction surviving through all routes) has averaged less than $33 \%$ for migration years 2007-2009 (Perry 2010).

Recovering endangered salmon populations in the Central Valley requires actions that mitigate the effects of water management on juvenile salmon. Increasing juvenile salmon survival in the Delta may consist of actions aimed at either reducing mortality within migration routes or directing the population away from low-survival migration routes such as the interior Delta. Quantifying potential benefits of implementing recovery actions can help fisheries managers weigh the costs of a given action against benefits measured in terms of increasing overall survival. In this study, we examine how altering migration routing can influence the overall survival of juvenile salmon.

In the Delta, migration routing of juvenile salmon can be altered in at least three ways. First, physical barriers, such as closure of the Delta Cross Channel gates, keep fish from entering a given migration route. However, physical barriers also alter the distribution of water flow, which can have unforeseen consequences on both fisheries and water resources. For example, closure of the Delta Cross Channel gates significantly alters the flows of many channels both upstream and downstream of the Delta Cross Channel, which in turn may affect entrainment and survival rates of multiple migration pathways. Closing the cross-channel gates can also increase the rate of salinity intrusion into the central Delta, ultimately reducing water exports in order to comply with mandated salinity standards. As this example shows, simply closing off a channel in the Delta is nontrivial, which has spurred investigation of alternative approaches for altering migration routing of salmon. For instance, non-physical behavioral barriers such as bubble curtains and strobe lights can elicit an avoidance response from juvenile salmon (Coutant 2001) while allowing water to flow unrestricted into a given channel. 
Fig. 1 Maps of the Sacramento-San Joaquin River Delta with shaded areas showing regions comprising survival through the Delta for four different migration routes. For each route, survival was estimated from Freeport on the Sacramento River (the northern most extent of the shaded area) to Chipps Island at the exit of the Delta (the western-most extent of the shaded area). In Route A, arrows show the two river junctions where migration routes diverge from the Sacramento River. For routes $\mathrm{C}$ and $\mathrm{D}$, the interior Delta is the large shaded region to the south of the Sacramento River. The location of the Delta Cross Channel is indicated by the arrow in Route C. The Sacramento River release site (off the map) is 19 river kilometers upstream of Freeport, and the Georgiana Slough release site is shown by the arrow in Route D

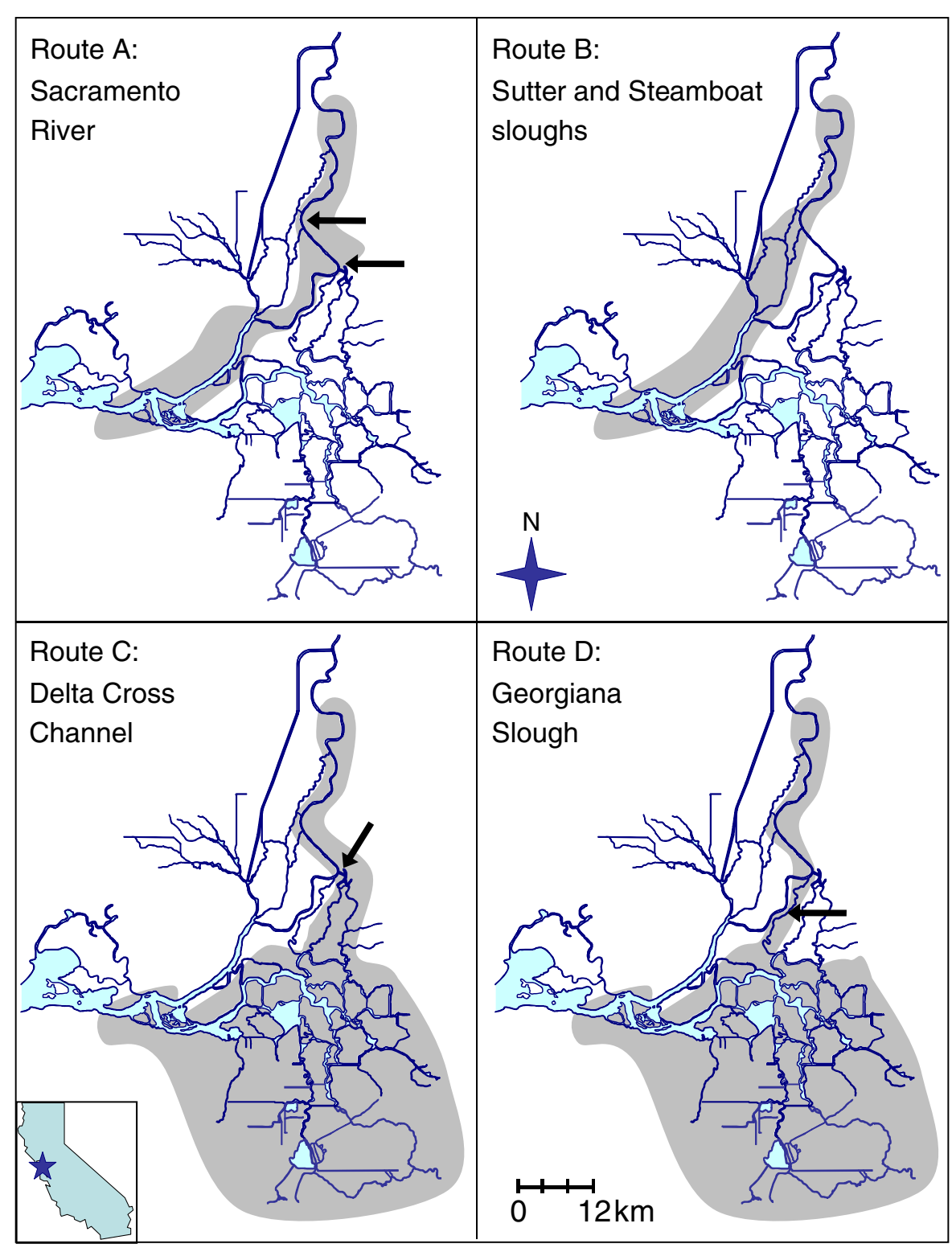

Finally, behavioral responses to the hydrodynamics at junctions may play a role in the entrainment rates at a given river junction. Thus, structural changes in channel geometry at a river junction may provide a means of altering migration routing without changing the distribution of water flow. Currently, both physical and non-physical behavioral barriers are being investigated in the Delta in attempt to guide fish away from low-survival migration routes.

To quantify the influence of migration routing on overall survival, we used estimates of movement and reach-specific survival obtained from acoustically tagged juvenile salmon collected over 3 years.
Biotelemetry techniques combined with markrecapture statistical models provide a powerful tool to simultaneously quantify dispersal and survival of juvenile salmon migrating through the Delta. Uniquely identifiable transmitters provided detailed information about the temporal and spatial movements of individuals migrating through a series of monitoring stations in the Delta. This information was then synthesized using a multistate mark-recapture model that quantified dispersal of the population among migration routes and survival within these routes (Perry et al. 2010). Simultaneously estimating these quantities allowed overall survival to be derived from each of 
these components. For this exercise, we examined the sensitivity of overall survival to migration routing by altering the distribution of fish at critical river junctions and then used the observed route-specific survival estimates to quantify how such actions would affect overall survival in the Delta.

\section{Methods}

To examine how migration routing influences overall survival of juvenile Chinook salmon in the Delta $\left(S_{\text {Delta }}\right)$, we used estimates of survival and routing probabilities provided by Perry et al. (2010) for the 2007 migration year and Perry (2010) for the 2008 and 2009 migration years. Route-specific survival, the fraction of fish migrating through each route, and overall survival were estimated from acoustic-tagged juvenile salmon using a multistate mark-recapture model applied to detection data from a system of telemetry stations situated throughout the Delta.

\section{Telemetry system}

Telemetry stations monitored movement of tagged fish among four primary migration routes through the Delta (Fig. 1): the mainstem Sacramento River (Route A); Sutter and Steamboat sloughs (Route B); the interior Delta via the Delta Cross Channel (Route C); and the interior Delta via Georgiana Slough (Route D). Each telemetry station consisted of single or multiple monitors (Vemco Ltd., Model VR2), depending on the number of monitors needed to maximize detection probabilities at each station. The number of telemetry stations varied among years (14, 23, and 20 stations in 2007,2008 , and 2009, respectively), but stations needed to estimate migration routing and survival to the terminus of the Delta remained constant among years. Detailed maps of the each year's telemetry system can be found in Perry (2010).

Fish tagging and release

Juvenile late fall Chinook salmon were obtained from and surgically tagged at the Coleman National Fish Hatchery in Anderson, California. For the first release in December 2006, a 1.44-g tag (Vemco Ltd., Model V7-1L-R64K, 40-d expected battery life) was used. For all other releases, we used a 1.6-g tag (Vemco
Ltd., Model V7-2L-R64K, 70-d expected battery life). Fish above $140 \mathrm{~mm}$ fork length were randomly selected for tagging. Transmitters were surgically implanted into fish using methods described by Perry et al. (2010).

To release tagged fish, they were first transported to release sites at either the Sacramento River near Sacramento, CA (all years) or Georgiana Slough (2008 and 2009; Fig. 1). The Georgiana Slough release site was added for 2008 and 2009 to increase the number of fish entering the interior Delta. In 2007 and 2008, fish were transferred to net pens (3-m square holding nets supported by pontoons) at the release site and held for $24 \mathrm{~h}$ in the Sacramento River prior to release to allow recovery from the transportation process. For 2009 , fish were transferred to perforated 121-L containers ( 2 fish per bucket) and held for $24 \mathrm{~h}$ in-river prior to release. Each release was carried out over a 24-h period to distribute tagged fish over the tidal and diel cycle. Two releases were performed in each migration year; one in December and another in January. For example, in migration year 2007, fish were released in December, 2006 when the Delta Cross Channel was open, and again in January, 2007 when the Delta Cross Channel was closed.

\section{Linking migration routing to overall survival}

The mark-recapture model described by Perry et al. (2010) estimates three sets of parameters: detection $\left(P_{h i}\right)$, survival $\left(S_{h i}\right)$, and route entrainment probabilities ( $\Psi_{h l}$; Perry 2010; Perry et al. 2010). Detection probabilities $\left(P_{h i}\right)$ estimate the probability of detecting a transmitter given a fish is alive and the transmitter operational at telemetry station $i$ within route $h(h=\mathrm{A}$, B, C, D). Survival probabilities $\left(S_{h i}\right)$ estimate the probability of surviving from telemetry station $i$ to $i+1$ within route $h$, conditional on surviving to station $i$. Route entrainment probabilities $\left(\Psi_{h l}\right)$ estimate the probability of a fish entering route $h$ at junction $l(l=1,2)$, conditional on fish surviving to junction $l$. Estimates of these parameters can be found in Perry (2010).

The first river junction was modeled as a twobranch junction where the entrance to Sutter and Steamboat Slough was pooled to estimate a single route entrainment probability. The parameter $\Psi_{\mathrm{B} 1}$ estimates the probability of being entrained into either Sutter or Steamboat Slough at the first river junction (Fig. 2). Conversely, $1-\Psi_{\mathrm{B} 1}=\Psi_{\mathrm{A} 1}$ is the probability 


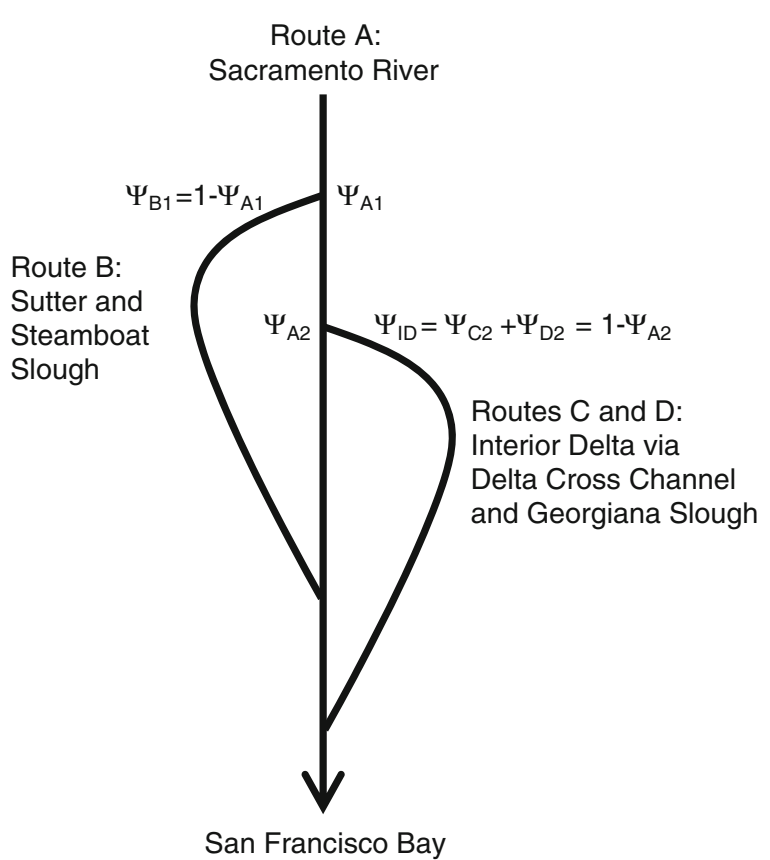

Fig. 2 Schematic showing the simplified routing structure and route entrainment probabilities $\left(\Psi_{h l}\right)$ at each river junction

of remaining in the Sacramento River at the first junction. The second junction was modeled as a three-branch junction where $\Psi_{\mathrm{A} 2}, \Psi_{\mathrm{C} 2}$, and $1-\Psi_{\mathrm{A} 2}-$ $\Psi_{\mathrm{C} 2}=\Psi_{\mathrm{D} 2}$ estimate the probabilities of remaining in the Sacramento River (Route A), being entrained into the Delta Cross Channel (Route C), and entering Georgiana Slough (Route D) at junction 2.

The mark-recapture model estimates the individual components that comprise survival of the population migrating through the Delta, defined as survival of tagged fish from the entrance to the Delta at Freeport (rkm 73) to the exit of the Delta at station Chipps Island (rkm -9), a distance of $82 \mathrm{~km}$ by way of the Sacramento River. Overall survival through the Delta was estimated from the individual components as:

$S_{\text {Delta }}=\sum_{h=\mathrm{A}}^{\mathrm{D}} \Psi_{h} S_{h}$

where $S_{h}$ is the probability of surviving the Delta given the specific migration route used to negotiate the Delta, and $\Psi_{h}$ is the probability of migrating through the Delta via one of four migration routes ( $\mathrm{A}=$ Sacramento River, $\mathrm{B}=$ Sutter and Steamboat sloughs, $\mathrm{C}=$ Delta Cross Channel, $\mathrm{D}=$ Georgiana Slough). Overall survival through the Delta is a weighted average of the route-specific survival probabilities with weights equal to the fraction of fish migrating through each route.

Migration route probabilities are a function of the route entrainment probabilities at each of the two river junctions:

$\Psi_{\mathrm{A}}=\Psi_{\mathrm{A} 1} \Psi_{\mathrm{A} 2}$

$\Psi_{\mathrm{B}}=\Psi_{\mathrm{B} 1}$

$\Psi_{\mathrm{C}}=\Psi_{\mathrm{A} 1} \Psi_{\mathrm{C} 2}$

$\Psi_{\mathrm{D}}=\Psi_{\mathrm{A} 1} \Psi_{\mathrm{D} 2}$

For instance, consider a fish that migrates through the Delta via the Delta Cross Channel (Route C). To enter the Delta Cross Channel, this fish first remains in the Sacramento River at junction 1 with probability $\Psi_{\mathrm{A} 1}$, after which it enters the Delta Cross Channel at the second river junction with probability $\Psi_{\mathrm{C} 2}$. Thus, the probability of a fish migrating through the Delta via the Delta Cross Channel $\left(\Psi_{\mathrm{C}}\right)$ is the product of these route entrainment probabilities, $\Psi_{\mathrm{A} 1} \Psi_{\mathrm{C} 2}$.

Survival through the Delta for a given migration route $\left(S_{h}\right)$ is the product of the reach-specific survival probabilities $\left(S_{h i}\right)$ that trace each migration path between the entrance to the Delta and its terminus at Chipps Island. Thus, $S_{h}$ is comparable among years even though annual differences in the telemetry system resulted in different reaches over which $S_{h i}$ was estimated. Furthermore, $S_{h}$ is directly comparable among routes because it estimates survival between the same starting and ending locations, but for fish migrating through different routes.

For our analysis, we focused on the probability of entering the interior Delta $\left(\Psi_{\mathrm{ID}}\right)$, which is the sum of the route entrainment probabilities for the Delta Cross Channel $\left(\Psi_{\mathrm{C} 2}\right)$ and Georgiana Slough ( $\Psi_{\mathrm{D} 2}$, Fig. 2). Survival through the interior Delta was estimated as the average survival of fish entering Routes $\mathrm{C}$ and $\mathrm{D}$, weighted by the entrainment probabilities for each route. We aggregated Routes C and D for this analysis because survival estimates for fish entering the interior Delta were consistently lower than other routes (Fig. 3) regardless of whether fish entered the interior Delta via the Delta Cross Channel or Georgiana Slough. Thus, the specific 


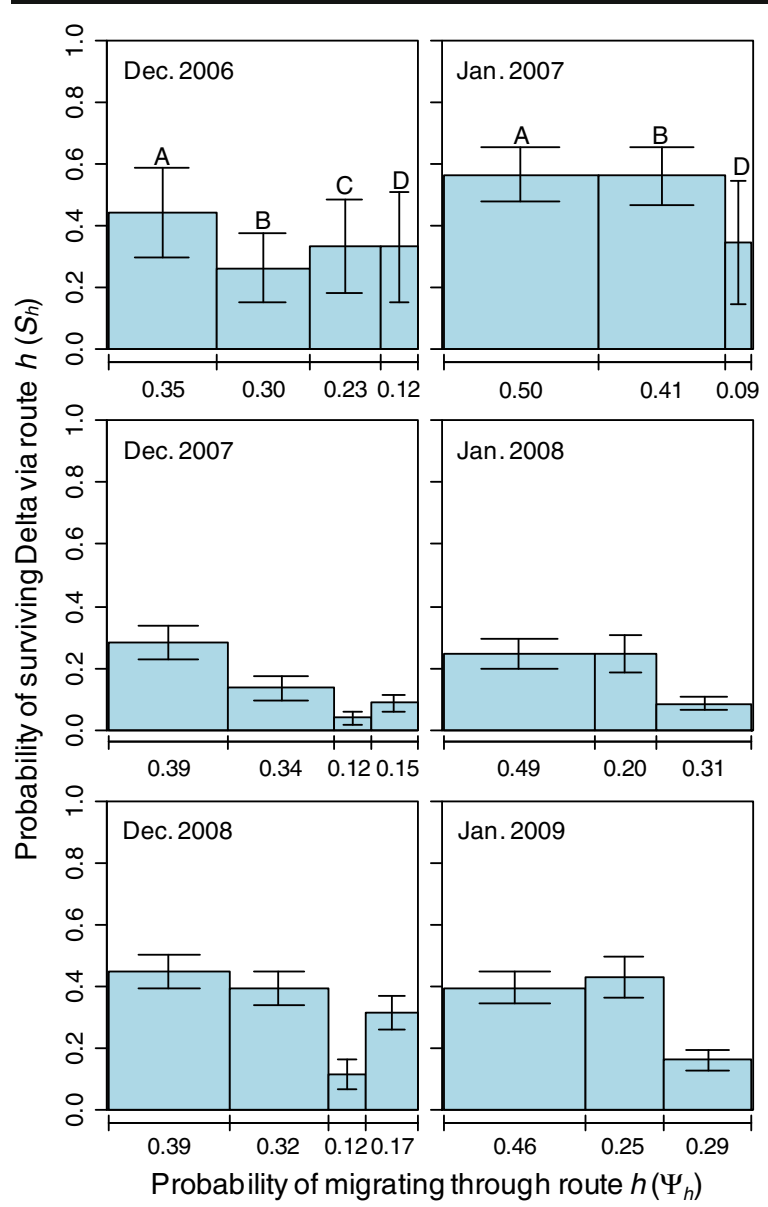

Fig. 3 Route-specific survival and the fraction of the population migrating through each migration route in the Sacramento San Joaquin River Delta (from Perry et al. 2010; Perry 2010). Migration routes are labeled as follows: $A=$ Sacramento River, $\mathrm{B}=$ Sutter and Steamboat sloughs, $\mathrm{C}=$ Delta Cross Channel, $\mathrm{D}=$ Georgiana Slough. Error bars show \pm 1 standard error

route used to enter the interior Delta is immaterial with respect to the sensitivity of overall survival to $\Psi_{\mathrm{ID}}$.

Influence of migration routing on $\mathrm{S}_{\text {Delta }}$

To quantify the influence of migration routing on $S_{\text {Delta, }}$, we examined the change in $S_{\text {Delta }}$ caused by varying route entrainment probabilities while holding constant the route-specific survival probabilities. Specifically, we examined the change in $S_{\text {Delta }}$ when varying 1) the probability of fish entering Sutter and Steamboat sloughs $\left(\Psi_{\mathrm{B} 1}\right)$, and 2$)$ the conditional probability of entering the interior Delta $\left(\Psi_{\mathrm{ID}}\right)$, given fish that remained in the Sacramento River at its junction with Sutter and Steamboat Slough (Fig. 2). For each release group, we varied entrainment probabilities between zero and one at each river junction, and then recalculated $S_{\text {Delta. }}$. We then quantified the predicted change in $S_{\text {Delta }}$ relative to the observed estimate of $S_{\text {Delta }}$ as both the absolute (i.e., additive) and relative (i.e., proportional) difference. This approach provides an understanding of how $S_{\text {Delta }}$ might have changed had survival probabilities been the same but migration routing different for each release group.

To understand the response of $S_{\text {Delta }}$ to changes in $\Psi_{h l}$, we also used demographic analysis techniques for matrix population models, which can be generalized to any transition matrix. For a Leslie matrix, sensitivity and elasticity measure the additive and proportional change in $\lambda$, the finite rate of population change, with respect to each demographic parameter in the model (Caswell 2001). In our case, $S_{\text {Delta }}$ is analogous to $\lambda$ in that it measures the rate of population change between the beginning and ending points of the Delta. Applying these techniques to our model, sensitivity is calculated as

$s_{\Psi_{h l}}=\frac{\partial S_{\text {Delta }}}{\partial \Psi_{h l}}$

and elasticity as

$e_{\Psi_{h l}}=\frac{\Psi_{h l}}{S_{\text {Delta }}} \frac{\partial S_{\text {Delta }}}{\partial \Psi_{h l}}$,

where $s_{\Psi_{h l}}$ and $e_{\Psi_{h l}}$ are sensitivity and elasticity with respect to a given route entrainment probability, $\Psi_{h l}$.

Sensitivity and elasticity can be interpreted in a number of ways to provide insights into how route entrainment probabilities affect $S_{\text {Delta }}$. First, sensitivity measures the slope of the relationship between absolute changes in $S_{\text {Delta }}$ and $\Psi_{h l}$, while elasticity measures the slope of proportional changes in $S_{\text {Delta }}$. The steeper the slope, the larger will be the effect on $S_{\text {Delta }}$ from a given change in $\Psi_{h l}$. Positive estimates indicate that increasing $\Psi_{h l}$ will increase $S_{\text {Delta, }}$, whereas negative values indicate that increasing $\Psi_{h l}$ will reduce $S_{\text {Delta }}$. Second, sensitivity and elasticity can be interpreted as the additive and proportional change in $S_{\text {Delta, }}$, respectively, when increasing $\Psi_{h l}$ from zero to one. For example, if $s_{\Psi_{\mathrm{ID}}}=-0.20$ then increasing $\Psi_{\mathrm{ID}}$ from zero to one will reduce $S_{\text {Delta }}$ by 20 percentage points (e.g., from 0.50 to 0.30 ). In contrast, $e_{\Psi_{\mathrm{ID}}}=$ -0.20 indicates a $20 \%$ change in $S_{\text {Delta }}$ (e.g., from 0.50 to 0.40 ). Last, applying Eq. 6 to $S_{\text {Delta }}$ Eq. 1 
yields a formula for the slope as a function of the route survival and entrainment parameters, elucidating which parameters affect the sensitivity of $S_{\text {Delta }}$ to route entrainment probabilities. Although differences in survival among routes will certainly influence sensitivity of $S_{\text {Delta }}$ to migration routing, sensitivity will also be a function of routing at both river junctions.

Last, we predicted $S_{\text {Delta }}$ by varying both $\Psi_{\mathrm{B} 1}$ and $\Psi_{\text {ID }}$ simultaneously to quantify the range in overall survival that could be obtained by altering entrainment at both river junctions. Such insights will help fisheries managers better understand how to target management actions aimed at altering route entrainment probabilities in order to maximize overall survival in the Delta.

\section{Results}

Interannual patterns in route-specific survival and migration probabilities

We observed substantial variation in the magnitude of within-route survival among years, yet stable patterns of survival across routes over all years (Perry 2010; Perry et al. 2010). Among migration years, 2008 stands out as having the lowest survival at both the route scale and the Delta scale (Fig. 3). Survival through the Delta was $<0.20$ for 2008 , but $>0.33$ for all other years and releases (Table 1). Over all years, estimates of $S_{\text {Delta }}$ exceeded 0.40 for only one release group (Jan. 2007), and only during migration year 2007 did observed estimates of $S_{\text {Delta }}$ differ considerably

Table 1 Sensitivity of $S_{\text {Delta }}$ to route entrainment probabilities for Sutter and Steamboat sloughs and the interior Delta. Also shown is sample size and estimates of $S_{\text {Delta }}$ for each release between releases (Table 1). For all releases, detection probabilities $\left(P_{h i}\right)$ were high at most sites (median $=1.0$, mean $=0.915$, minimum $=0.385)$, leading to favorable precision of survival probabilities relative to releases sample sizes (Table 1, Fig. 3).

Although rankings of route-specific survival vary somewhat across release groups, one pattern remained consistent: survival probabilities for the Sacramento River were always greater than survival for migration routes through the interior Delta (via Georgiana Slough and the Delta Cross Channel; Fig. 3). In addition, Sutter and Steamboat sloughs exhibited either similar survival to the Sacramento River (typically for January releases) or lower survival than the Sacramento River (typically for December releases; Fig. 1). Except for the Dec. 2007 release group, observed survival estimates for Sutter and Steamboat Sloughs were greater than for routes leading to the interior Delta.

Sensitivity of $S_{\text {Delta }}$ to route entrainment probabilities

The effect of varying route entrainment probabilities on overall survival differed among river junctions. At the first river junction, sensitivity of $S_{\text {Delta }}$ to entrainment into Sutter and Steamboat Slough $\left(\Psi_{\mathrm{B} 1}\right)$ followed no consistent trend among releases. Increasing $\Psi_{\mathrm{B} 1}$ decreased $S_{\text {Delta }}$ for two of the releases, increased it for two releases, and resulted in a slight positive change in $S_{\text {Delta }}$ for two releases (Table 1; Fig. 4a, b). In addition, the standard errors for sensitivity and elasticity of $\Psi_{\mathrm{B} 1}$ indicate that the $95 \%$ confidence intervals overlap

group (from Perry et al. 2010, Perry 2010). Standard errors are given in parentheses and were based on variances estimated using the Delta method

\begin{tabular}{|c|c|c|c|c|c|c|}
\hline \multirow[t]{2}{*}{ Release group } & \multirow[t]{2}{*}{ Number released } & \multirow[t]{2}{*}{$S_{\text {Delta }}$} & \multicolumn{2}{|c|}{ Sutter and Steamboat Slough, $\Psi_{\mathrm{B} 1}$} & \multicolumn{2}{|c|}{ Interior Delta, $\Psi_{\mathrm{ID}}=\Psi_{\mathrm{C} 2}+\Psi_{\mathrm{D} 2}$} \\
\hline & & & Sensitivity & Elasticity & Sensitivity & Elasticity \\
\hline Dec. 2006 & 64 & $0.351(0.101)$ & $-0.125(0.116)$ & $-0.105(0.098)$ & $-0.078(0.123)$ & $-0.111(0.175)$ \\
\hline Jan. 2007 & 80 & $0.543(0.070)$ & $0.030(0.101)$ & $0.023(0.077)$ & $-0.129(0.126)$ & $-0.036(0.038)$ \\
\hline Dec. 2007 & 208 & $0.174(0.031)$ & $-0.059(0.042)$ & $-0.117(0.085)$ & $-0.142(0.038)$ & $-0.331(0.085)$ \\
\hline Jan. 2008 & 211 & $0.195(0.034)$ & $0.062(0.051)$ & $0.063(0.052)$ & $-0.127(0.041)$ & $-0.252(0.073)$ \\
\hline Dec. 2008 & 292 & $0.368(0.037)$ & $0.038(0.058)$ & $0.033(0.050)$ & $-0.148(0.045)$ & $-0.170(0.053)$ \\
\hline Jan. 2009 & 292 & $0.339(0.035)$ & $0.125(0.071)$ & $0.093(0.054)$ & $-0.176(0.044)$ & $-0.200(0.054)$ \\
\hline
\end{tabular}


zero for all release groups (95\% confidence interval=estimate $\left.\pm 1.96^{*} \mathrm{SE}\right)$. In contrast, at the second river junction, increasing entrainment into the interior Delta $\left(\Psi_{\mathrm{B} 1}\right)$ decreased $S_{\text {Delta }}$ for every release group, and the confidence intervals for four of the six releases exclude zero (Table 1; Fig. 4c, d).

Changes in $S_{\text {Delta }}$ in response to migration routing at a given junction are driven partly by differences in survival among migration routes and partly by entrainment probabilities at other river junctions. For example, for the two releases where $S_{\text {Delta }}$ declined when increasing $\Psi_{\mathrm{B} 1}$ (Dec. 2006 and Dec. 2007; Fig. 4a, b), the negative slope was driven by lower survival in Sutter and Steamboat Sloughs than in the Sacramento
River (Fig. 3). For all other releases, survival was similar between the Sutter and Steamboat Sloughs (Route B) and the Sacramento River (Route A, Fig. 3), yet $S_{\text {Delta }}$ responded positively to increasing the proportion of fish entering Sutter and Steamboat Slough (Table 1; Fig. 4a, 4a). Examining the equation for sensitivity of $S_{\text {Delta }}$ with respect to $\Psi_{\mathrm{B} 1}$ reveals why this pattern emerges:

$s_{\Psi_{\mathrm{B} 1}}=\left(S_{\mathrm{B}}-S_{\mathrm{A}}\right)+\Psi_{\mathrm{ID}}\left(S_{\mathrm{A}}-S_{\mathrm{ID}}\right)$.

The first term shows that sensitivity is partly a function of the difference in survival between the Sacramento River and Sutter and Steamboat sloughs $\left(S_{\mathrm{B}^{-}}\right.$ $\left.S_{\mathrm{A}}\right)$. However, the second term in the equation shows
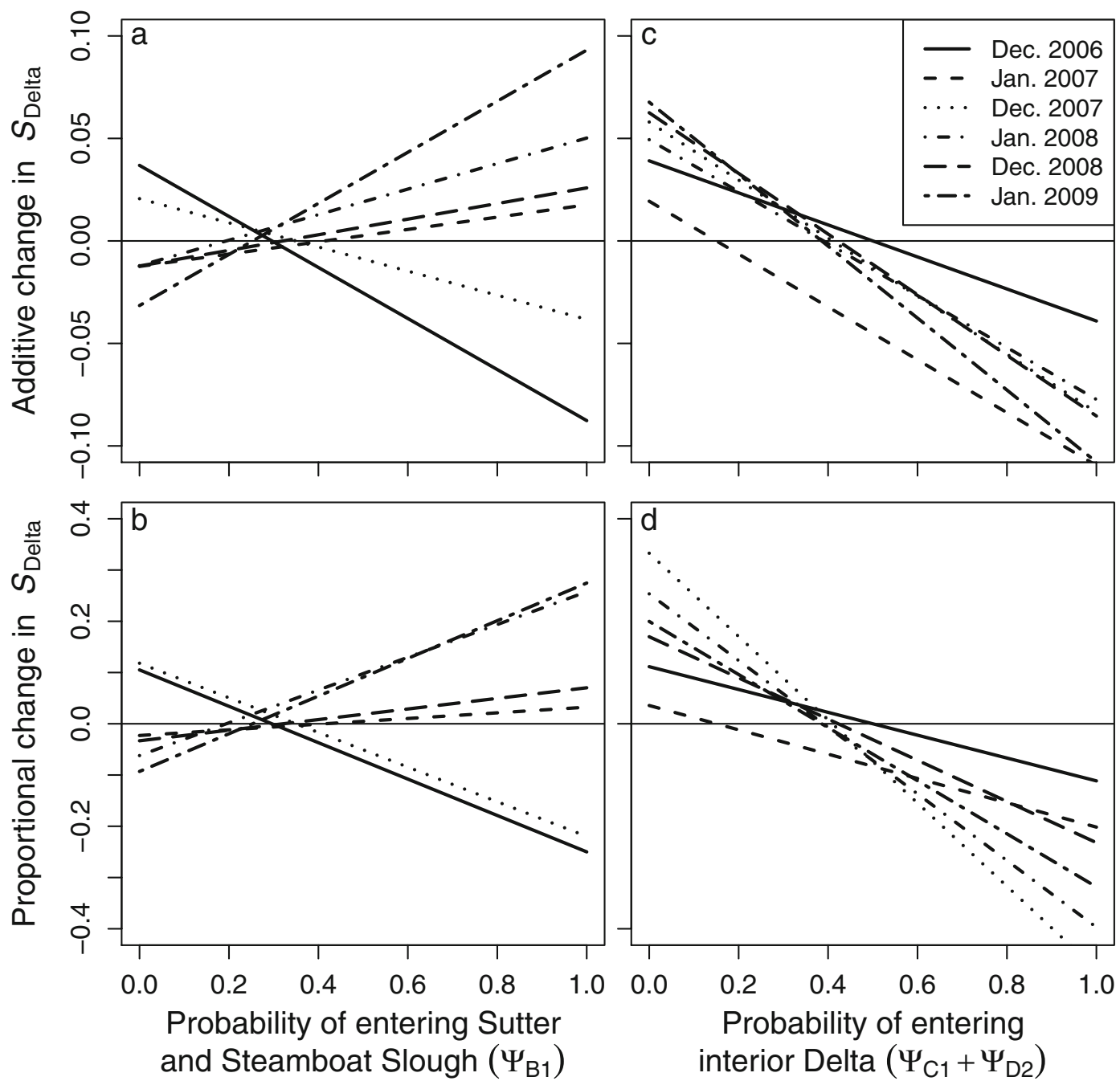

Fig. 4 Predicted change in estimates of $S_{\text {Delta }}$ for each release group in response to varying route entrainment probabilities between zero and one 
that sensitivity is also driven by 1 ) the probability of entrainment into the interior Delta $\left(\Psi_{\mathrm{ID}}\right)$, and 2$)$ the difference in survival between the Sacramento River and interior Delta $\left(S_{\mathrm{A}}-S_{\mathrm{ID}}\right)$. Consequently, when survival for Sutter and Steamboat sloughs is on par with the Sacramento River $\left(S_{\mathrm{B}}-S_{\mathrm{A}} \approx 0\right)$, increasing $\Psi_{\mathrm{B} 1}$ increases $S_{\text {Delta }}$ by routing fish away from the interior Delta where survival was lower than the Sacramento River.

At the second river junction, increasing entrainment into the interior Delta always reduced $S_{\text {Delta }}$ because survival for the interior Delta (Routes C and D) was lower than the Sacramento River (Route A) for all release groups (Fig. 3). However, the magnitude of change in $S_{\text {Delta }}$ depends on not only differences in survival between these routes, but also on the fraction of the population remaining in the Sacramento River at the first river junction:

$s_{\Psi_{\mathrm{ID}}}=\Psi_{\mathrm{A} 1}\left(S_{\mathrm{ID}}-S_{\mathrm{A}}\right)$.

Although the difference in survival between these routes determines the direction of change in $S_{\text {Delta, }}$, $\Psi_{\mathrm{A} 1}$ scales the magnitude of change. For example, for the Jan. 2009 release group, survival of fish entering the interior Delta was 0.235 less than the Sacramento River (i.e., $S_{\mathrm{ID}}-S_{\mathrm{A}}=0.163-0.398$ ). But because $25 \%$ of the tagged population entered Sutter and Steamboat Slough at the first river junction (Fig. 3), the maximum possible change in $S_{\text {Delta }}$ is only 0.175 when changing $\Psi_{\text {ID }}$ from one to zero (Table 1). These findings illustrate how the magnitude of change in $S_{\text {Delta }}$ from altering entrainment at one river junction depends not only on differences in survival between alternative routes, but also on the fraction of the population passing the river junction.

Eliminating entrainment into the interior Delta is expected to result in a 2-7 percentage point increase in overall survival (Fig. 4c). As discussed above, the magnitude of this change is, in part, due to only a fraction of the tagged population passing by this river junction. However, the small absolute increase in survival is also due to low survival probabilities observed in all routes. Route-specific survival for all routes was $<0.5$ for most release groups (Fig. 3). Thus, while shifting the distribution of fish among routes influences overall survival, the magnitude of absolute change in $S_{\text {Delta }}$ is constrained by maximum survival observed in any given route. Further increases in $S_{\text {Delta }}$ would require management actions that affect not only migration routing, but also survival within migration routes.

In contrast, proportional changes in $S_{\text {Delta }}$ provide insight into the relative change in survival in response to altering route entrainment probabilities. $S_{\text {Delta }}$ varied considerably among years (Table 1 ) even though relative differences in survival between the Sacramento River and interior Delta remained consistent among years (Fig. 3). Therefore, given interannual variation in overall survival, proportional changes in $S_{\text {Delta }}$ allow comparison among release groups on a common relative scale. From this perspective, the relative change in $S_{\text {Delta }}$ is considerably larger than the absolute change, increasing by $10-35 \%$ for five of the six releases in response to eliminating entrainment into the interior Delta. This analysis shows how understanding changes in $S_{\text {Delta }}$ on both absolute and relative scales is important, particularly when overall survival is low and varies through time.

Altering entrainment at both river junctions simultaneously revealed that 1 ) overall survival could vary considerably in response to migration routing, 2) the optimal strategy for maximizing survival varied among releases, and 3) sensitivity of overall survival to entrainment at one junction depended the value of entrainment at the other river junction. Depending on release group, maximum $S_{\text {Delta }}$ was 1.5 to 2.4 times the minimum survival (Fig. 5). Although survival can be maximized simply by directing fish to the highest-survival route, the set of entrainment probabilities that maximize survival varied among release groups. For December releases, since the Sacramento River (Route A) exhibited higher survival than other routes, overall survival is maximized when all fish remain in the Sacramento River (i.e., when $\Psi_{\mathrm{B} 1}=0$ and $\Psi_{\mathrm{ID}}=0$; Fig. 5). However, for January release groups, overall survival is maximized by minimizing entrainment into the interior Delta but maximizing entrainment in Sutter and Steamboat Slough. Because survival in the Sacramento River was similar to Sutter and Steamboat Slough during January releases, diverting fish into Sutter and Steamboat Slough maximizes overall survival by routing fish away from the second river junction where they become exposed to entering the interior Delta.

Simultaneously altering entrainment probabilities at both river junctions illustrated how sensitivity of $S_{\text {Delta }}$ to entrainment at one junction depends on the value of entrainment at the other river junction (Fig. 5). Vertical 
Fig. 5 Contour plot showing overall survival through the Sacramento - San Joaquin River Delta $\left(S_{\text {Delta }}\right)$ predicted as a function of entrainment probabilities into the interior Delta and Sutter and Steamboat Slough. Filled circles show the observed overall survival and route entrainment probabilities for each release group

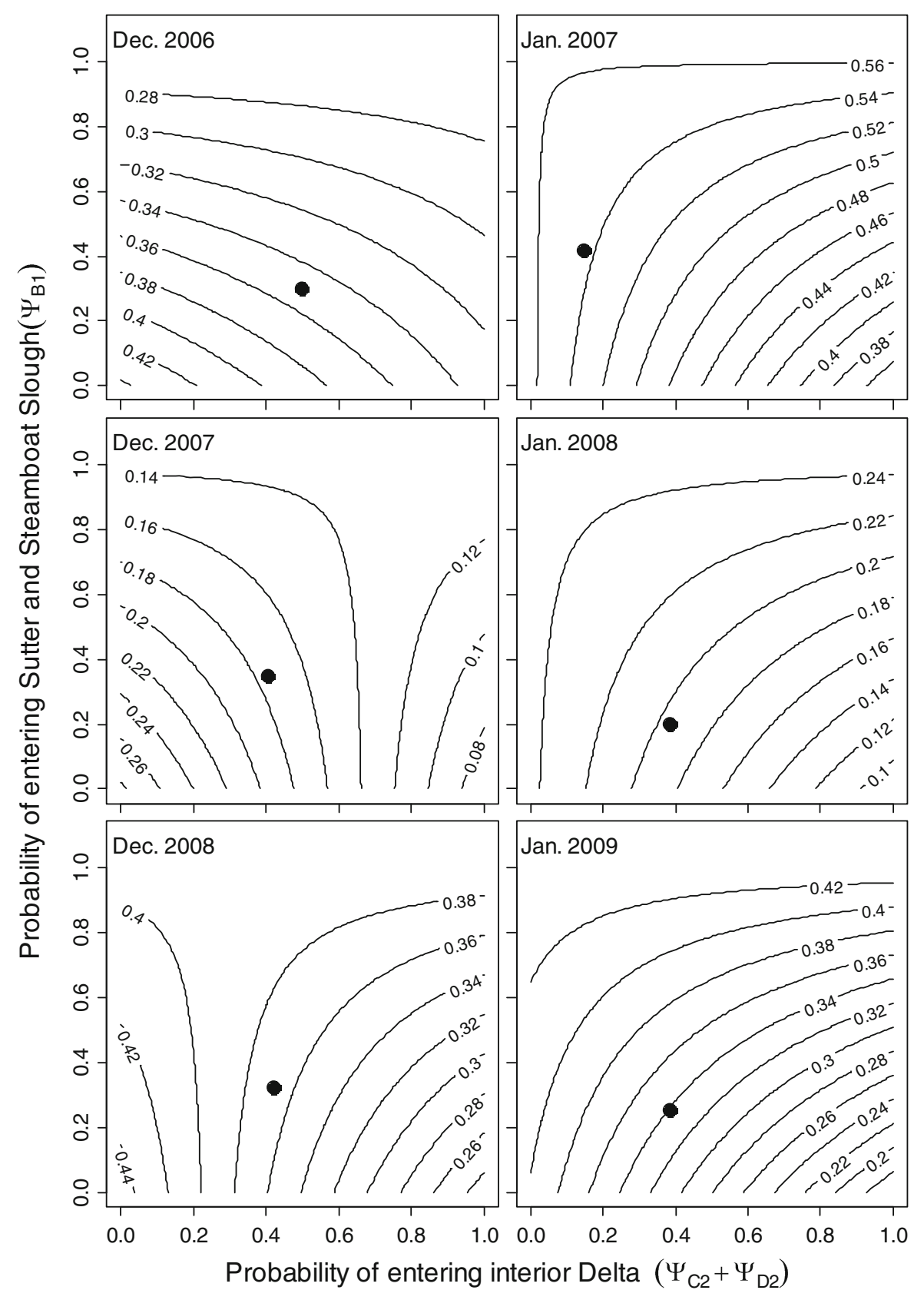

contour lines in Fig. 5 indicate regions where $S_{\text {Delta }}$ is insensitive to $\Psi_{\mathrm{B} 1}$, horizontal contour lines reveal insensitivity to $\Psi_{\mathrm{ID}}$, and closely-spaced contour lines reveal regions of high sensitivity. For example, as entrainment into Sutter and Steamboat Slough increases, $S_{\text {Delta }}$ becomes less sensitive to changes in $\Psi_{\text {ID }}$ because most of the population is diverted away from the second river junction. For January releases, $S_{\text {Delta }}$ is insensitive to $\Psi_{\mathrm{B} 1}$ when $\Psi_{\mathrm{ID}}$ is low, as is indicated by the wide range of $\Psi_{\mathrm{B} 1}$ that yields similar overall survival. These relationships help to understand how survival through Delta varies in response to migration routing.

\section{Discussion}

Our analysis reveals the magnitude of change in overall survival that might be expected from management 
actions that alter migration routing through the Delta. Given the substantial difference in survival between the interior Delta and the Sacramento River, we might have expected a larger boost in survival from eliminating entrainment into the interior Delta. In a simpler system with only one branching junction (e.g., a dam), change in overall survival with respect to migration routing is directly proportional to the difference in survival among migration routes. However, due to the channel complexity of the Delta, altering migration routing at one river junction yields changes in $S_{\text {Delta }}$ that are less than proportional to the difference in survival between alternative migration routes. We showed that changes in $S_{\text {Delta }}$ with respect to migration routing at one river junction depends also on migration routing at other river junctions. Therefore, by considering how management actions at multiple river junctions affect $S_{\text {Delta, }}$ managers may be able to optimize the suite of actions required to maximize the expected increase in $S_{\text {Delta. }}$. These are important insights about the magnitude of increase in $S_{\text {Delta }}$ expected from management actions to alter migration routing.

The strength of inferences from acoustic tag data to the untagged population depend on whether survival estimates are viewed from a relative or absolute point of view. Potential tag effects on survival (Adams et al. 1998) or differences in survival between hatchery and wild fish (Reisenbichler and McIntyre 1977; Kostow 2004) could result in lower absolute survival of tagged fish relative to untagged fish. In our study, although it is unknown whether tagged fish of hatchery origin exhibit lower survival than untagged fish of wild origin, absolute changes in survival should be interpreted with caution (i.e., Fig. 4a, c). Regardless of the absolute magnitude of survival, however, differences among routes that influence survival should act similarly on all populations of salmon smolts migrating through the Delta. For example, both tagged and untagged fish migrating through the interior Delta likely experienced lower survival relative to fish migrating within the Sacramento River. Therefore, relative changes in survival in response to altering migration routing (i.e., Fig. $4 c$, d) should provide stronger inferences to untagged populations than will absolute change in survival probabilities.

We focused our analysis on river junctions where management actions are likely to have the largest influence on population survival. For example, we showed that Steamboat and Sutter Slough is an important migration route because fish using this route avoid entering the interior Delta where survival is lower than other routes. The Delta's channel geometry is hierarchical in nature such that secondary (and finer level) migration routes are nested within primary routes. At each secondary and tertiary river junction, the population divides into a smaller and smaller fraction of the whole. Therefore, management actions focused at secondary junctions will have less population-level influence than at primary river junctions simply because a small fraction of the population will be influenced. In contrast, management actions have the potential for influencing much of the population at the two primary river junctions examined in our analysis.

Sensitivity and elasticity measure changes in $S_{\text {Delta }}$ with respect to migration routing at a junction while holding all other parameters constant. Thus, our analysis assumes that management actions alter only migration routing but not route-specific survival probabilities. This assumption may be violated in two ways. First, changing migration routing will alter the abundance of juvenile salmon in each route, which could cause a density dependent predator response. At very low prey densities, increasing smolt abundance within a route could increase predation rates via the predator's numerical or functional response to prey. In contrast, increasing smolt abundance to high levels within a route could reduce predation rates through predator swamping. Second, management actions that affect water routing at a particular junction (e.g., physical barriers) could influence route-specific survival or entrainment at other junctions by changing discharge and hydrodynamics within a migration route. For example, physical barriers alter discharge entering each channel, and juvenile salmon survival has been positively correlated with discharge in the Delta (Newman and Rice 2002; Perry 2010). Such simultaneous changes in migration routing and routespecific survival are not captured by our analysis.

In terms of the magnitude of change in population survival, managers must consider both the expected change in migration routing and the expected change in route-specific survival caused by implementation of physical and non-physical barriers. With respect to migration routing, physical barriers are $100 \%$ effective whereas non-physical barriers typically divert less than $100 \%$ of fish. Therefore, under the assumption of constant route-specific survival, non-physical barriers would realize only a fraction of the maximum possible increase in population survival. With respect to route-specific survival, physical barriers may yield 
a larger change in survival than non-physical barriers because physical barriers alter discharge and hydrodynamics of each migration route. However, the direction and magnitude of change in route-specific survival in response to physical and non-physical barriers is poorly understood. This uncertainty highlights the importance of quantifying simultaneous changes in both migration routing and route-specific survival in field studies evaluating physical and non-physical barriers in the Delta.

Our sensitivity analysis has application to other regulated river systems where managers must balance the costs of water management actions against benefits to fish populations. On the Columbia River, for example, millions of dollars are spent annually to evaluate survival of juvenile salmon migrating past dams. Management actions such as spilling water over dams results in foregone power generation but improves population survival of juvenile salmon by diverting them away from turbines. Our analytical approach could be used to quantify expected changes in population survival by implementing such actions, helping managers to better design dam operations to achieve recovery targets at minimum cost. More importantly, in the Delta and other regulated river systems, our analytical approach can be used to help design recovery actions before such actions are implemented. Given scarce resources with which to recover endangered salmon populations, such analyses can help direct resources towards actions most likely to yield the largest improvement in survival.

Acknowledgements Funding for R.W.P's involvement with this project was provided by a CALFED Science Fellowship, Agreement No. U-04-SC-005 with the California Bay-Delta Authority. Tagging of juvenile salmon, ultrasonic station deployment and interrogation, and tag-detection database maintenance were supported by a grant from the California Bay-Delta Authority by Agreement No. U-05-SC-047. We thank the staff of Coleman National Fish Hatchery for providing the late-fall Chinook and logistical support for this study. Staff of the Stockton U.S. Fish and Wildlife Office gratefully assisted with fish transportation and release. We thank two anonymous reviewers for comments that substantially improved this manuscript.

\section{References}

Adams NS, Rondorf DW, Evans SD, Kelly JE, Perry RW (1998) Effects of surgically and gastrically implanted radio transmitters on swimming performance and predator avoidance of juvenile Chinook salmon (Oncorhynchus tshawytscha). Can J Fish Aquat Sci 55:781-787

Bracis C (2010) A model of the ocean migration of Pacific salmon. Thesis, University of Washington

Brandes PL, McLain JS (2001) Juvenile Chinook salmon abundance, distribution, and survival in the Sacramento-San Joaquin Estuary. In: Brown RL (ed) Contributions to the biology of Central Valley salmonids, volume 2, Fish Bulletin 179. California Department of Fish and Game, Sacramento, California, pp 39-138

Caswell H (2001) Matrix population models: construction, analysis, and interpretation. Sinauer Associates, Inc., Sunderland, Massachusetts

Coutant CC (2001) Behavioral technologies for fish guidance. American Fisheries Society, Bethesda, Maryland

Kostow KE (2004) Differences in juvenile phenotypes and survival between hatchery stocks and a natural population provide evidence for modified selection due to captive breeding. Can J Fish Aquat Sci 61:577-589

Newman KB, Brandes PL (2010) Hierarchical modeling of juvenile Chinook salmon survival as a function of Sacramento-San Joaquin Delta water exports. N Am J Fish Manage 30:157-169

Newman KB, Rice J (2002) Modeling the survival of Chinook salmon smolts outmigrating through the lower Sacramento River system. J Am Stat Assoc 97:983-993

Perry RW (2010) Survival and migration dynamics of juvenile Chinook salmon (Oncorhynchus tshawytscha) in the Sacramento-San Joaquin River Delta. Dissertation, University of Washington

Perry RW, Brandes PL, Sandstrom PT, Ammann A, MacFarlane B, Klimley AP, Skalski JR (2010) Estimating survival and migration route probabilities of juvenile Chinook salmon in the Sacramento-San Joaquin River Delta. N Am J Fish Manage 30:142-156

Reisenbichler RR, McIntyre JD (1977) Genetic differences in growth and survival of juvenile hatchery and wild steelhead trout, Salmo gairdneri. J Fish Res B Can 34:123-128

Skalski JR, Townsend R, Lady J, Giorgi AE, Stevenson JR, McDonald RD (2002) Estimating route-specific passage and survival probabilities at a hydroelectric project from smolt radiotelemetry studies. Can J Fish Aquat Sci 59:1385-1393

Skalski JR, Buchanan RA, Townsend RL, Steig TW, Hemstrom $S$ (2009) A multiple-release model to estimate routespecific and dam passage survival at a hydroelectric project. N Am J Fish Manage 29:670-679 